\title{
A Quantum Neurological Model of Perception-Cognition and Awareness in Ambiguous Figures and the Case of the Dalmatian Dog
}

\author{
Elio Conte ${ }^{1 *}$, Ignazio Licataa,2,3, Raúl Alelú-Paz ${ }^{4,5}$ \\ ${ }^{1}$ School of Advanced International Studies for Applied Theoretical and Non Linear Methodologies of Physics, \\ Bari, Italy \\ ${ }^{2}$ ISEM, Institute for Scientific Methodology, Palermo, Italy \\ ${ }^{3}$ B.M. Birla Science Centre, International Institute for Applicable Mathematics \& Information Sciences (IIAMIS), \\ Hyderabad, India \\ ${ }^{4}$ Laboratory for Neuroscience of Mental Disorders Elena Pessino, Department of Medicine and \\ Medical Specialties, School of Medicine, Alcalá University, Madrid, Spain \\ ${ }^{5}$ Department of Psychiatry, Ramón y Cajal Hospital, Instituto Ramón y Cajal de Investigación Sanitaria (IRYCIS), \\ Madrid, Spain \\ Email: "elio.conte@fastwebnet.it, ignazio.licata@ejtp.info, raul.alelu@uah.es
}

Received 3 October 2015; accepted 21 November 2015; published 24 November 2015

Copyright $@ 2015$ by authors and Scientific Research Publishing Inc.

This work is licensed under the Creative Commons Attribution International License (CC BY).

http://creativecommons.org/licenses/by/4.0/

(c) (i) Open Access

\section{Abstract}

The present paper has mainly the aim to formulate a quantum neurological model of brain dynamics during perception and cognition of ambiguous figures here including the case of the Dalmatian dog. First of all, we summarize our previous experimental and theoretical studies, evidencing as the basic conceptual foundations of quantum mechanics enter in a decisive manner in explaining the basic functions of brain dynamics at perceptive and cognitive level and at the level of our consciousness. This is done in detail in Sections 2 and 3 where we discuss also the role of the so called quantum interference effect and we include here the results of an our recent experiment confirming the role of quantum theory and of its quantum interference effect also at perceptive-cognitive and awareness levels. In Section 4, following our previous study, we consider the so called Mach-Zehnder interferometer. It is a physical device that is employed in quantum mechanical experiments in order to analyze such quantum interference effect. Soon after the discussion of this device, we proceed with the elaboration of a detailed quantum neurological model in the sense that we consider the brain regions identified in V1; V2/V3/V4 and A20 and we obtain that they may behave just as the beam splitters involved in the Mach-Zehnder quantum mechani-

*Corresponding author.

How to cite this paper: Conte, E., Licata, I. and Alelú-Paz, R. (2015) A Quantum Neurological Model of Perception-Cognition and Awareness in Ambiguous Figures and the Case of the Dalmatian Dog. Journal of Behavioral and Brain Science, 5, 533549. http://dx.doi.org/10.4236/jbbs.2015.512051 
cal interferometer. In this manner, we formulate a model of V1; V2/V3/V4, A20 brain dynamics as a Mach-Zehnder interferometer and such model delineates a proposed neurological mechanism explaining perception-cognition and awareness in humans under the input of ambiguous figures and in the particular case of the Dalmatian dog.

\title{
Keywords
}

\author{
Ambiguous Figures, Dalmatian dog, Mach-Zehnder Interferometer, Quantum Cognition, Quantum \\ Interference, Quantum Consciousness
}

\section{Introduction}

In recent decades, neuroscientists have tried to solve the so-called mind-body problem from different disciplines, developing experimental research in healthy human subjects, in neurological or psychiatric patients, and in some animal models [1]. Their findings suggest that the conscious world that appears to the viewer is the result of complex processes of integration of signals from multiple sensory modalities performed by the cortex. In this respect, visual phenomenal consciousness is by far the most experimentally investigated aspect. This sense allows us to obtain vast information about different aspects of our world, such as the spatial dimensions of our surroundings or the emotions of others. As humans are mainly visual animals, a prime interest in neuroscience revolves around understanding the function of the visual system, including different aspects such as cognition, the neural substrates serving the visual sense and developing physical models of visual information processing. Although tremendous efforts have been made to explain how the brain is able to generate a conscious visual percept, we are still far from a scientific explanation. The reason is clear: consciousness poses special characteristics that are not encountered in other domains of science, that is, to connect a description of brain processes with our own individual experience. In this regard, we consider crucial to try to identify the physical laws that could underlie the neurological processes resulting in the emergence of consciousness. In this regard, quantum mechanics has made different proposals [2]-[4]. Some focused on the function of quantum processes in the microtubules of neurons [5], the analysis of quantum coherent oscillations in neuronal signaling [6] or the development of a quantum mechanical model to explain conscious perception during binocular rivalry [7], the relationship between scale relativity and fractal medium structures with Quantum Potential [8] [9] among others. Starting with 1983 and particularly by theoretical and experimental studies that we initiated in 2003 and currently continued until today, we investigated the possible role of quantum mechanics analyzing the possibility that mental states follow quantum mechanics during perception and cognition. We studied in particular the ambiguous figures, the Stroop effect, some cognitive anomaly as the conjunction fallacy, the priming effect, the integration of emotions and cognition in children. We give a preliminary list of some results here [10]-[38].

Let us sketch briefly the status of the matter.

Presently brain sciences are going through a very important conflict. From one hand, we have neuroscience. This discipline is giving every day new basic knowledge also thanking neuro-imaging contributions. Unexpected fundamental results neuroscience is giving constantly. However, in spite of such relevant results, it does not arrive to give the most expected result that is to explain the actual nature of mental entities. On the other hand, we have the empirical results of the psychology. Also in this case, we have constant new contributions but often they result conflicting with those of neuroscience. In this way, the situation is that in the middle between neuroscience and psychology there is the darkness that has to be overcome by the presence of a bridge that should be represented by a new model. Quantum mechanics is not a possible candidate but the basic foundation to represent this model not by speculations, not by epistemological considerations, not for tentative and attempts but because the science of quantum mechanics demonstrates that this is the case. This is the scientific background that legitimates the advent of quantum cognition.

In order to reach the objective to cover the dark gap existing presently between neurological contributions from one hand and psychological empirical results from the other hand, we need to elaborate detailed formulations where first of all we identify the basic neurological mechanisms and consequently we delineate detailed and convincing quantum neurological models explaining the involved mechanisms. In order to follow this line, we started in 2011 [15] [16] where for the first time we introduced the Mach-Zehnder interferometer to explain 
perception and cognitive function in brain.

Using such interferometer approach, in the present paper we present, for the first time, a quantum model explaining the mechanism of perception and cognition at a neurological level in ambiguous figures, trying to bridge the gap existing between both scientific approaches. In the following section, we summarize the basic results we obtained previously in this field to finally give a detailed explanation of the quantum neurological model proposed here.

\section{Introductory Remarks on the Role of Quantum Mechanics in Perception and Cognition in Humans}

Perception is the mechanism by which our brain captures stimuli and information arising from our external or inner material and non-material world. According to Jansen [22] [39] extra-mental reality can only be perceived by the brain with the help of our sense organs which transmit all information with the help of physical factors from extra-mental reality, such as electromagnetic waves for the eyes or other physical factors for all other sense organs. Such sense organs transform these factors into neural activity, an integrative process called sensory transduction that allows the brain to construct a mental representation of extra-mental reality. Light enters the eye as electromagnetic waves and stimulates sensory neurons in the retina, which transforms the physical stimulation into depolarization of neurons and transmits their information to specialized brain regions. Mechanical waves enter the ear and stimulate specialized receptors, which transmit their activation to specialized regions in other brain regions. All sensory organs function in a similar way, since they are stimulated by physical factors and transmit their activity to their corresponding special brain regions.

The key is in the following basic statement: with information perceived by all sensory organs, the brain constructs a mental representation of extra-mental reality.

Let us continue with Jansen's elaboration: if the perception organs are inactivated, i.e. by closing the eyes or the ears, there is no longer any direct physical contact between the extra-mental reality and its mental representation in the brain, hence, no observation of extra-mental physical events is still possible. We can say that the brain, in this situation, is isolated from its outside environment and works only with its memory, that is, with past perceptions encoded and retrieved. The distinction between sensory perception through direct physical contact with the extra-mental reality and memorized perceptions of the past is very important, since pure bottom-up perception from sensory organs to the brain remain unchanged, but not its cognitive aspects; for example, the intensity of powerful light flashes or intolerable noise which cannot be voluntarily changed, whereas perceptions of the same events after their memorization become modifiable (i.e. forgotten or recalled a day later) and no longer induce the same feelings of pain. Since these memorized past perceptions can be cognitively modified, they allow rearrangements in the representation of extra-mental reality by arranging new situations, which no longer correspond to prior perceived extra-mental reality. Therefore, these functions are essential for arranging the future by reorganizing past memory perceptions in a different way and projecting them in the mind into the future, which is, in turn, generally indeterminate; hence can only be arranged with potentiality or, in other words, by performing simultaneous superposition of possible alternatives happening in accord to a field of probabilities. We have to repeat here the basic statement: since the future is generally constructed in our mind with indetermination, it can only be arranged with potentiality and this term means by performing simultaneous superposition of possible alternatives happening in accord to a field of probabilities. The potentiality realized by simultaneous superposition of intrinsically indeterminate alternatives and the connected field of probabilities, regulating such intrinsic indetermination of the existing alternatives, directly connect the structure of the abstract mental entities around which our brain functions, as repeatedly outlined in the past from Margenau and Eccles [13] [40] [41]. So we have to consider several alternatives or possibilities existing simultaneously-although with different probabilities - which correspond, in humans, to mental superposition for the prediction of an unknown future.

Brain has different peculiar features, the most original one is that it constantly attempts to realize predictions about the future and in such operation brain moves with intrinsic indetermination.

Let us consider that a given mental condition admits two alternatives (i.e. an input that gives indetermination to a subject in its understanding). It may be perceived and understood as $|+\rangle$ or $|-\rangle$ before to reach a final decision as it happens as example in the case of Figure 1.

The subject is consequently posed in a condition of intrinsic and contextual indetermination-a mental statethat emerges as a result of a potential simultaneous superposition of two alternatives: $\mid$ Mental State $\rangle=c_{1}|+\rangle+c_{2}|-\rangle$ 


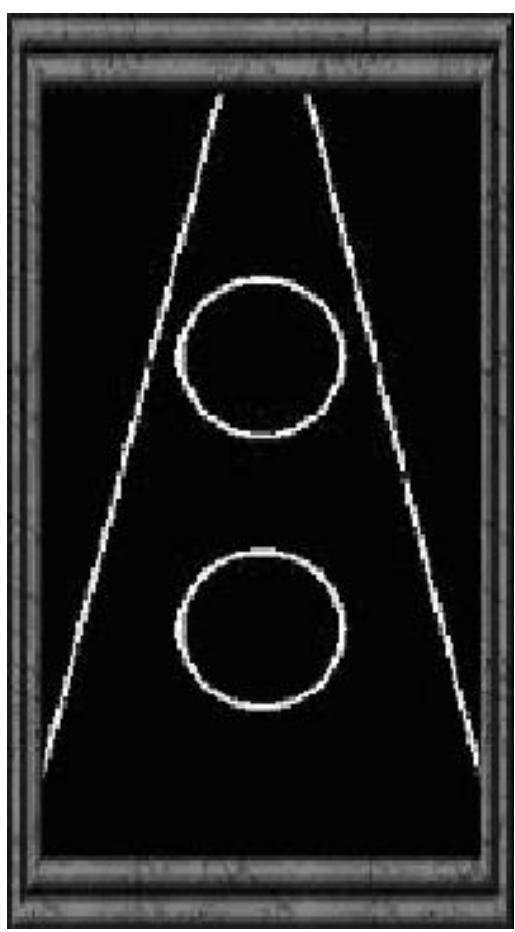

Figure 1. Are the two circles equal?

being $c_{1}$ and $c_{2}$ probability amplitudes. The square modulus $\left|c_{1}\right|^{2}$ and $\left|c_{2}\right|^{2}$ give the probabilities that, being the subject under such condition of simultaneous superposition of alternatives with intrinsic indetermination, soon after, will, at conscious level, decide to select alternative $|+\rangle$ or $|-\rangle$.

As mentioned above, every time the subject is posed in a condition of intrinsic indetermination and, therefore, will realize a mental state whose counterpart is a quantum superposition of alternatives and, finally, at perceptive and cognitive level, will select one of the two alternative probabilistically with a dynamics that is dependent from the context in which it operates. Consequently, quantum probability field is the abstract mental entity regulating our decisional processes.

Therefore, according to this principle of quantum superposition, the mental state of the subject realizes for a certain time a condition of intrinsic indetermination and a simultaneous superposition of alternatives.

In detail in the case of Figure 1 we write:

$$
\left.\left.\mid \text { Mental State }\rangle=c_{1} \mid \text { equal circles }\right\rangle+c_{2} \mid \text { not equal circles }\right\rangle
$$

Following such superposition within a given time, the subject with a certain probability will select only one of the two alternative: he/she will decide and his/her mental state will be

$$
\mid \text { the two circles are equal }\rangle \text { or } \mid \text { the two circles are not equal }\rangle
$$

His/her initial perceptive cognitive function of AND will translate in the final perceptive-cognitive condition of OR in his/her consciousness.

In summary,

a) After a linear superposition of different alternatives, the subject will consciously perceives and cognitively deliberate about one and only one between the two potential outcomes.

b) $\mathrm{He} / \mathrm{she}$ will deliberate with a certain contextually dependent probability.

In quantum mechanics, we define the collapse of the wave function as the acting mechanism that induces such AND/OR transitions and the basic importance of our formulation resides in the fact that we must be able to evidence that our consciousness responds just to such basic quantum mechanical foundations and, in particular, to the superposition quantum principle and connected rules of probabilities. 
In order to provide such evidence we have to introduce some considerations about the mechanism of perception. As explained in detail elsewhere [18] [19] [22] the human eye shows high levels of sensitivity, even to perceive a single photon. But not all the inputs reach consciousness; to avoid visual noise, perception filters make a selection that begins in the retina, specifically at the cone and rod levels. The former are responsible for color vision, being much less sensitive to low light than the rods and showing a high activity in bright light (photopic vision). When we enter a dark room, the eyes first adapt by opening up the iris to allow more light in. Over a period of about 30 minutes, other chemical adaptations make the rods become sensitive to light at about a 10,000 th of the level needed for the cones to work. After this time we see much better in the dark, but we have very little colour vision (scotopic vision). Different authors have tested this phenomenon: Hecht, Schlaer and Pirenne [42] concluded that the rods could respond to a single photon during scotopic vision while Baylor, Lamb and Yau [43] were able to use toads' rods placed into electrodes to show directly that they respond to single photons. Finally, our group recently published that binocular vision and/or binaural hearing raise interesting explanations at the level of consciousness [18] [19] [22]. The active substance in the rods is rhodopsin. A single photon can be absorbed by a single molecule that changes shape and chemically triggers a signal that is transmitted to the optic nerve. It is possible to test our visual sensitivity by using a very low level light source in a dark room.

As Woo outlined just in 1981 [44], since the stimuli that the two eyes receive, are compared in the brain to yield depth and motion, since the vision of split brain subjects shows clear left-right differences, we may conclude that we are subconsciously aware, some of time, of which eye sees which. This is the central point. In fact, it follows immediately that we may perform an experiment in a dark room. We may arrange the signal so that it reaches one and only one of our eyes. In the case, we are normally unaware which eye has actually seen the signal. In this condition we are forced to acknowledge that there is a state of the consciousness which corresponds to a coherent superposition of seeing the signal by the right eye and seeing the same signal by the left eye. As previously outlined the rods must be responding to single photons; since the light quantum coming into the left eye would excite the left retina (which excitation can in turn be checked), it would seem that even in principle there can be no interference between the wave function $\psi_{r}$ corresponding to seeing a flicker by excitation of the right retina. A similar reasoning may be developed by us for $\psi_{l}$. We have not so much alternatives in the arising conclusion. The whole visual system is so tightly correlated that there exists a state of awareness $\psi_{0}$ which is a coherent superposition of $\psi_{r}$ and $\psi_{l}$ with the same energy for the total system.

The unquestionable conclusion is that consciousness responds to the rules of quantum mechanics.

\section{On some Further Confirmations That Quantum Mechanics Holds at Perceptive-Cognitive and Awareness Levels}

As previously stated, in recent years our group has focused on the analysis of the role of quantum mechanics during perception and cognition, conducted studies on the Stroop effect, cognitive anomalies (such as the conjunction fallacy), ambiguous figures, priming, and recently on the integration between cognition and emotion in children.

Our results demonstrate an existing quantum interference effect that is peculiar only of quantum mechanics. Briefly, we give a sensory input to a Group A of subjects by using a task that admits as answer a dichotomous variable $A$, taking the possible two values, +1 or -1 . The probabilities $p(A=+1)$ and $p(A=-1)$ are then estimated. A second group of subjects (Group $B$ ) are involved in a different task, introducing a new variable $B$ with possible values $B=+1$ or $B=-1$, before submitting them to the task already proposed to Group $A$. In this case, the following probabilities may be estimated $p(B=+1), \quad p(B=-1), \quad p(A=+1 / B=+1)$, $p(A=+1 / B=-1), p(A=-1 / B=+1)$, and $p(A=-1 / B=-1)$.

If we do not apply quantum mechanics during the perceptual and cognitive processes of subjects submitted to the tasks, classical Bayes's theorem must hold and we should obtain,

$$
p(A=+1)(\operatorname{group} A)=p(B=+1) p(A=+1 / B=+1)+p(B=-1) p(A=+1 / B=-1)(\text { group } B)
$$

and a similarly expression for $p(A=-1)$.

On the contrary, if we include in our analysis the superposition principle and quantum interference, confirming the role for quantum mechanics during the perception and the cognition, we have 


$$
\begin{aligned}
p(A=+1)= & p(B=+1) p(A=+1 / B=+1)+p(B=-1) p(A=+1 / B=-1) \\
& +2 \sqrt{p(B=+1) p(A=+1 / B=+1) p(A=-1 / B=+1)} \cos \vartheta
\end{aligned}
$$

which implies the presence of the quantum interference term

$$
2 \sqrt{p(B=+1) p(A=+1 / B=+1) p(A=-1 / B=+1)} \cos \vartheta
$$

and a similarly expression for $p(A=-1)$.

We face a critical turning point; we have the possibility to discern if we are in presence of a mental dynamics in which quantum mechanics has a basic role or, on the contrary, can be explained without it. If quantum mechanics has a role we have that the formula containing the quantum interference term is verified by experimental data. If instead quantum mechanics has not a role we will verify that the classical Bayesan formula is verified.

In the present paper, we are concerned with the use of ambiguous figures. When we see an ambiguous figure the brain makes a bet; as example, Figure 1, in which the subject has to decide if the two circles are equal or not. We have a dichotomic variable A that may assume the value +1 if the subject in the end deliberates that the two circles are equal, and assumes instead the value -1 if the subject deliberates that the two circles are not equal. Usually, we make an immediate, context dependent choice (transition potentiality/actualization, collapse of the wave function) and see it in either one way or the other. In detail, we uphold that it is important to return to recall the quantum foundations that we outlined previously: the fact that upon continued exposure the interpretation often oscillates back and forth, leads to the basic evidence that at some point the state of consciousness realizes the quantum superposition that we have previously recalled. This is precisely the quantum superposition of the two modes that correspond to the different interpretations. It is the quantum superposisition of the potential alternatives that we have previously explained. They give interference effects arising from the two modes. This is what in conclusion our experiments have always confirmed. First we have a quantum superposition of states, that is to say, a quantum superposition of potential alternatives, the previous AND condition that we have mentioned, followed soon after from a transition to OR ( the collapse of the wave function) in which only one of the two coexisting alternatives is realized.

As further confirmation of our approach, we have here the possibility to introduce the results of an experiment that we have recently conducted: a subject looks at a screen on which the ambiguous picture reported in Figure 1 is flashed. It has an intrinsic ambiguity that leads to intrinsic indetermination and thus to quantum superposition of potential mental states. $\left(1^{+}\right)$and $\left(1^{-}\right)$. For the sake of simplicity we have arranged the experimental context in a particular experimental condition respect to the experiments that we performed previously: we selected only normal subjects chosing the two alternatives with equal probability. We have $p(1+)=0.5, p\left(1^{-}\right)=0.5$. After a short time interval, we give to the subjects a second ambiguous figure with possible interpretations (2+) or $(2-)$. We have in this case conditional probabilities $p(2+/ 1+)$. Algebraically we may express it by $\operatorname{sen}^{2} \alpha$. The probability of chosing (2+) if the first choice was (1-) is $p\left(2+/ 1^{-}\right)$and we may express it by $\operatorname{sen}^{2} \beta$. Note that we may determine $p\left(2+/ 1^{+}\right)$and $p\left(2+/ 1^{-}\right)$experimentally and in our experiments we obtain respectively $p\left(2+/ 1^{+}\right)=$ 0.35 and $p(2+/ 1-)=0.45$.

Now we consider a fresh group of test subjects submitted to the first ambiguous figure followed by exposure to the second ambiguous figure and we estimate the probability $p(2+)$.

For the details of the experiment we mention here that we studied 300 normal subjects, 150 females and 150 men with age ranging from 25 to 30 years. According to the classical Bayesan approach we should obtain for $p(2+)$ the following value

$$
\frac{p(2+/ 1+)+p(2+/ 1-)}{2}=\frac{\operatorname{sen}^{2} \alpha+\operatorname{sen}^{2} \beta}{2}=0.40
$$

and instead we obtain the value 0.60 .

The conclusion is that the classical probability rules are violated in this experiment. Quantum probability rules hold. Quantum mechanics has a role in this experiment. In detail, the two interpretations, that are the two alternatives of the first ambiguous figure, coexist (quantum mechanical superposition) in the consciousness of the subjects and the two modes interfere in the subsequent interaction of the subject with the second ambiguous figure. 


\section{On the Elaboration of a Neurological Quantum Model at Perceptive and Cognitive Level}

We have previously evidenced the particular role of quantum interference and, now, we must attempt to explicate that at the neurological level and at perceptive level we can formulate a detailed quantum neurological model based on quantum interference that allows us to explain brain dynamics when using an ambiguous figure.

In order to reach this objective we have to re-examine some foundations of quantum mechanics with the finality to characterize in detail the model of reality that it delineates and how much it is distant from our traditional manner of conceiving it.

Let us start discussing a device (see Figure 2).

In the device represented in Figure 2, we can see an orange marble. Represented in blue, there are two equal separators with the property that $50 \%$ of the times, the marble passes straight and in the other $50 \%$, it deviates the marble (randomly). In red, we have two barriers which direct the marble towards the second separator heavenly. In dark blue, we represent the possible trajectories of the marble and, in green and yellow, we have two containers that collect them. Let us examine what we expect that it happens during this experiment and what are instead the unexpected true results. We throw many marbles, making sure that the next ball is not thrown before the previous one has arrived at one of the two containers. Note that the path length is equal in both the cases. According to our intuition a marble after the first separator has a $50 \%$ of probability to pass straight. When it reaches the second separator, the marble has $50 \%$ of probability ending up in the yellow container and 50\% in the green container (overall 25\% will go to the yellow container and 25\% to the green one). The same is true even if the marble takes the horizontal path after the first separator, that is to say, it has $25 \%$ of probability ending up in the yellow container and $25 \%$ in the green one.

So throwing many balls, always one at a time, our expectations of finding them in the yellow container will be $25 \%+25 \%=50 \%$ and in the green container of $25 \%+25 \%=50 \%$.

Instead, according to quantum mechanics and performing the experimental verification of our expectations, we discover a strange result: no marble is finite in the yellow container, but all in the green.

Let us sketch what happens according to quantum mechanics.

In the so called Mach-Zehnder interferometer we have five elements (see Figure 3 and Figure 4).

We have a source that emits a particle at a time, two separators semitransparent beam (called Beam Splitter, or BS), two mirrors that deflect the direction of propagation of the particles, two detectors and a "differentiator" walk (see the Figure 3 and Figure 4). The particles that protrude from the source are microscopic, for example photons or neutrons.

We go through the interferometer many particles. We know that the beam splitters are balanced, that is, a particle that arrives at the first beam splitter has $50 \%$ of probability of continue straight and $50 \%$ to be deflected downward. Intuitively we can think that half reach the detector D1 and the other half reach the detector D2.

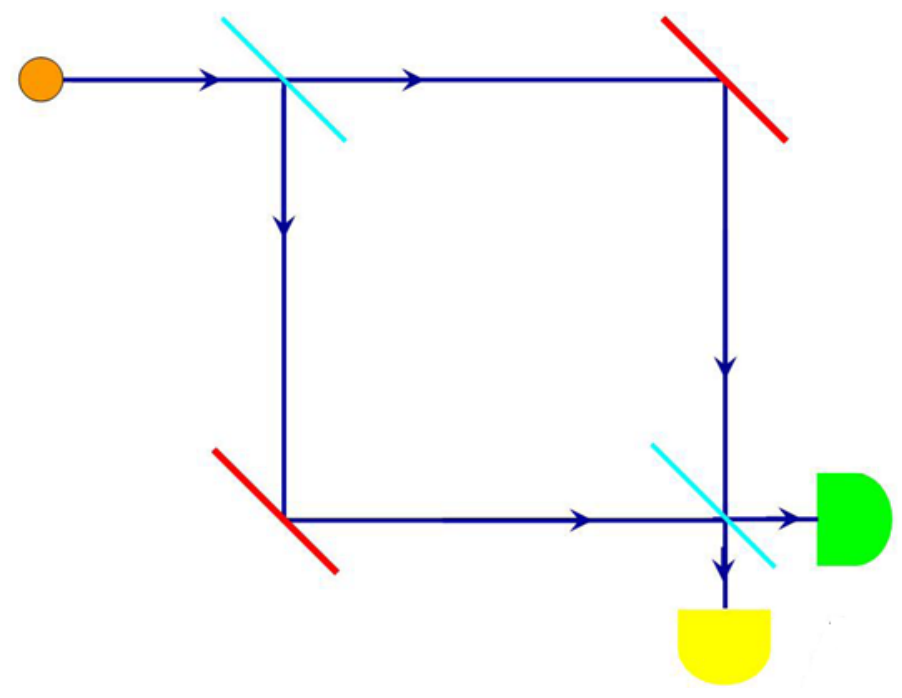

Figure 2. A rough scheme of the Mach-Zehnder interferometer. 


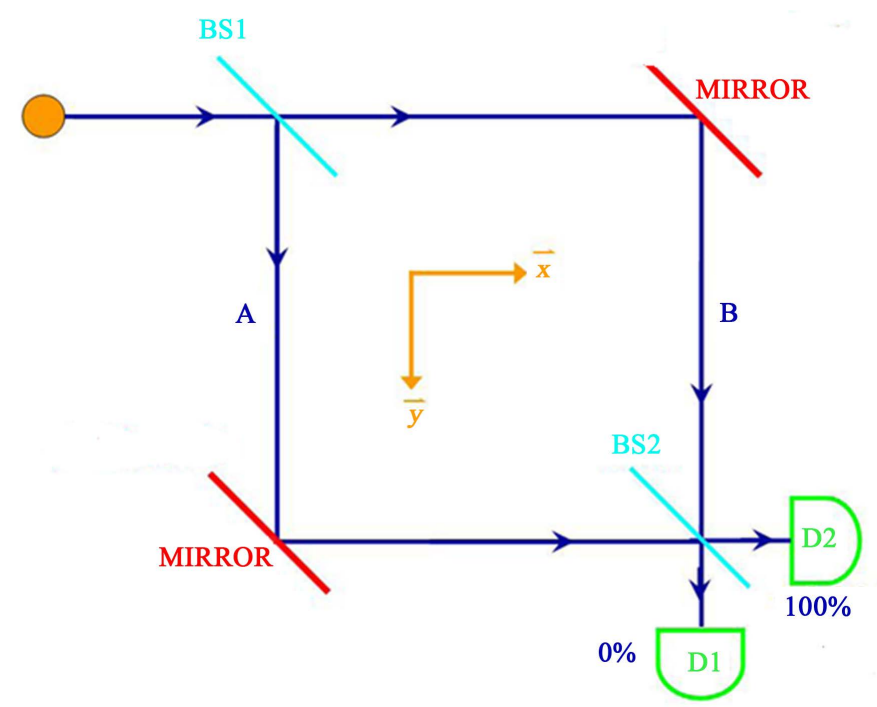

Figure 3. The Mach-Zehnder interferometer.

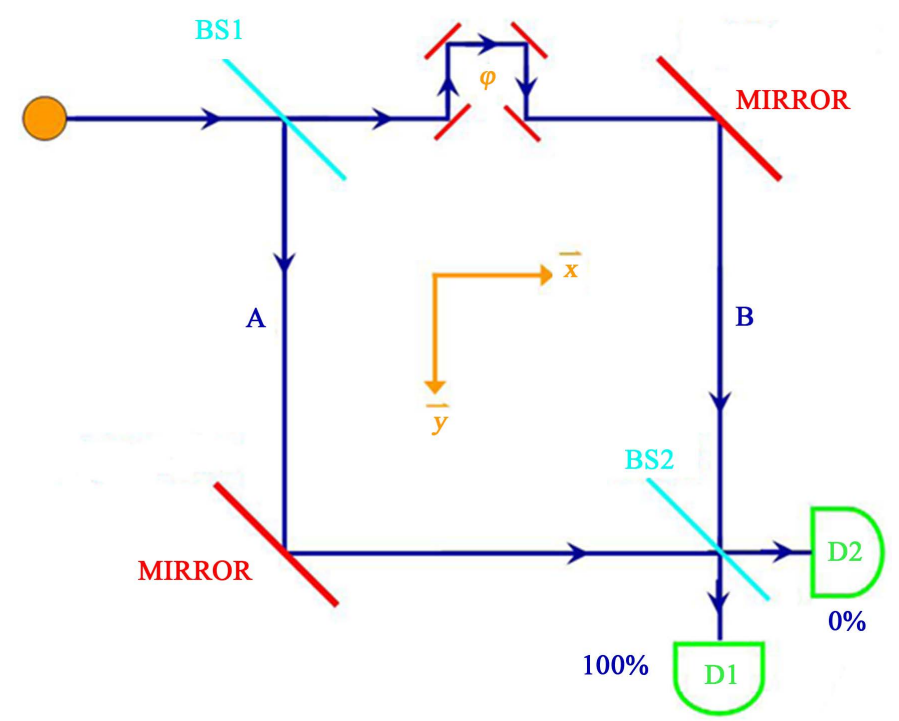

Figure 4. The Mach-Zehnder interferometer with modified pattern.

Quantum mechanics and experimental verifications demonstrate us that this never happens: all particles are detected in D1 and not in D2.

Since we have been through a lot of particles through the interferometer, we can say (classically) that surely some will be passed from the path A and other from the path B to get to the BS2. At this point we repeat the experience but with a variant: introduce a suitable variation of the path on the way $B$, as shown in Figure 4. In this case, all the particles are detected in D2, that is, the opposite situation to the previous one (without modification of the path).Yet we have introduced the amendment on an only path (B), and then at least the particles (in the first experiment should be passed from path A to arrive at the detector D1) should not have been affected by the change included in B; so we should expect to find some particles also in D1. Since the interferometer passes one particle at a time we can also exclude that we had effects due to collective bumps or other.

Therefore we have to conclude that the change of pattern in B also influences the pattern in A.

We have to give the explanation. We have a system with two alternatives. The possible results with the detectors are two: the particle is detected (which attribute the value 1 ) or not (value 0 ).

Since to each detector we associate the possible results (1) or (0), we have 
D2: $X=\left(\begin{array}{ll}1 & 0 \\ 0 & 0\end{array}\right) ; \mathrm{D} 1: Y=\left(\begin{array}{cc}0 & 0 \\ 0 & 1\end{array}\right)$

$X$ is the quantum observable connected to the device D2 that is able to detect in accord to the direction $x$ while instead $Y$ is the quantum observable connected to the device D1 according to the direction $y$.

On this point let us make a detailed statement.

In accord with our precedent studies on quantum cognition and, in particular on those performed by using the Mach-Zehnder interferometer, we have to highlight that quantum theory has its origin in the logic and thus in cognition rather than in matter. We obtained a first demonstration using logic statements and emphasizing that they are subjected to quantum interference as well as matter. The second demonstration was obtained just by using the Mach-Zehnder interferometer that we are using in the present paper [15]. We concluded on the logical origins of quantum mechanics and about the basic foundation that, according to such results, it is not true the thesis that matter and mental entities (i.e. perception and cognition) exist in our reality independently. We concluded that there are stages of our reality in which we cannot conceive "matter per se" that is to, independently from cognition we have about it [15]-[17] [20] [45] [46]. When matter is engaged (as example light targeting our eyes), we have to consider simultaneously the physical process in itself and the mental perceptive and the cognitive function that of course are simultaneously activated. The Mach-Zehnder interferometer involves not only a physical device but also a detailed cognitive scheme.

This is the basic result that we will apply in the present paper.

We have introduced here the observables $X$ and $Y$. We have to outline here that, using Clifford algebra, they are the basic idempotents

$$
X=\frac{1+e_{3}}{2} \text { and } Y=\frac{1-e_{3}}{2}
$$

These are idempotents in Clifford algebra and as such they represent logic statements. Logic statements imply the direct role of the cognition.

Let us indicate the states on $x$ and on $y$ respectively as

$$
\psi_{x}=\left(\begin{array}{l}
1 \\
0
\end{array}\right) \text { and } \psi_{y}=\left(\begin{array}{l}
0 \\
1
\end{array}\right)
$$

as it is immediate to obtain by application of $X$ on $\psi_{x}$ and $Y$ on $\psi_{y}$

The initial state (before the particle reaches BS1) is indicated by us obviously by $\psi_{x}$.

Therefore we have $\psi_{\text {in }}=\psi_{x}$.

The state after BS1 is obviously a quantum state of superposition that we have discussed in detail previously. Consequently we write

$$
\psi_{\text {out }}=\alpha \psi_{x}+\beta \psi_{y}
$$

where $\alpha$ and $\beta$ are probability amplitudes with $|\alpha|^{2}$ and $|\beta|^{2}$ the two corresponding probabilities as previously explained.

In order to estimate the $\psi_{\text {out }}$ we have first of all to estimate the pattern of the state and this is obtained in quantum mechanics by application of the Pattern Evolution Operator on $\psi_{\text {in }}$ relating the BS so that we write

$$
\psi_{\text {out }}=U_{B S} \psi_{\text {in }}
$$

where

$$
U_{B S}=\left(\begin{array}{ll}
a & b \\
c & d
\end{array}\right)
$$

We write $\psi_{\text {out }}$ in the following explicit manner

$$
\psi_{\text {out }}=\sqrt{t} \psi_{x}+\sqrt{r} \mathrm{e}^{i \phi} \psi_{y}
$$

where we have considered $\alpha=\sqrt{t} \mathrm{e}^{i \varphi_{1}}$ and $\beta=\sqrt{r} \mathrm{e}^{i \varphi_{2}}$ and $\varphi=\varphi_{2}-\varphi_{1}$.

Considering 


$$
\psi_{\text {out }}=U_{B S} \psi_{\text {in }}
$$

we finally obtain

$$
U_{B S}=\left(\begin{array}{cc}
\sqrt{t} & i \sqrt{1-t} \\
i \sqrt{1-t} & \sqrt{t}
\end{array}\right)
$$

that is still the Clifford member $U_{B S}=\sqrt{t}+i \sqrt{1-t} e_{1}$

The mirrors are no more than a particular case of the BS obtained by posing $r=0$.

We have now all the elements to estimate the final state $\psi_{\text {out }}$.

When the pattern is modified we have

$$
\psi_{\text {out }}=\mathrm{e}^{\mathrm{i} \varphi} \psi_{\text {in }}
$$

and the pattern evolution operator is

$$
U=\left(\begin{array}{cc}
\mathrm{e}^{\mathrm{i} \varphi} & 0 \\
0 & 0
\end{array}\right)
$$

that is still the Clifford member

$$
U=\mathrm{e}^{\mathrm{i} \varphi}\left(\frac{1+e_{3}}{2}\right)
$$

In conclusion we have the final state as pattern evolution of the initial state

$$
\psi_{\text {fin }}=U_{\text {fin }} \psi_{\text {in }}
$$

and following the pattern of the particle we have that $U_{\text {fin }}$ will be given from the first BS passage followed from the passage by the mirror, followed still by the modified pattern (phase) and finally by BS. In conclusion we have that

$$
U_{\text {fin }}=U(\text { BS2 }) U(\text { mirror }) U(\text { phase }) U(\text { BS1 })
$$

with

$$
U_{\text {fin }}=-\frac{1}{2}\left(\begin{array}{cc}
1+\mathrm{e}^{i \varphi} & -i\left(1-\mathrm{e}^{\mathrm{i} \varphi}\right) \\
i\left(1-\mathrm{e}^{i \varphi}\right) & 1+\mathrm{e}^{\mathrm{i} \varphi}
\end{array}\right)
$$

that is still the Clifford member.

$$
U_{f i n}=-\frac{1}{2}\left(\left(1+\mathrm{e}^{i \varphi}\right)+\left(1-\mathrm{e}^{i \varphi}\right) e_{2}\right)
$$

The final state may be calculated as

$$
\psi_{\text {fin }}=U_{\text {fin }} \psi_{\text {in }}
$$

and we obtain

$$
\psi_{\text {fin }}=-\mathrm{e}^{\mathrm{i} \varphi / 2} \cos (\varphi / 2) \psi_{x}+\mathrm{e}^{\mathrm{i} \varphi / 2} \operatorname{sen}(\varphi / 2) \psi_{y}
$$

and the probabilities to find the particle in D1 or in D2 are respectively

$$
\operatorname{Prob}(Y=1)=\frac{1}{2}(1-\cos \varphi)
$$

and

$$
\operatorname{Prob}(X=1)=\frac{1}{2}(1+\cos \varphi)
$$

The results are now in perfect accord with the experimental verification. 
If the phase $\varphi=0$ is equal to zero, all the particles will be in D2 while instead if the phase is $\varphi=\pi$ all the particles will be in D1. For intermediated values of $\varphi$, we have probabilities of finding particles in D1 and D2.

This is confirmed in all from the experimental results.

We must necessarily introduce a brief note of clarification. In the preceding lines, we have made extensive use of developments in mathematics and physics, and we understand that this development could lead to difficulties in understanding in scholars who have not direct mathematical and physical competence. We have done this for rigor exposure. However, the reader is interested only in the evaluation of the probabilities that the marble reaches the two containers and then the reader can safely ignore the mathematical and physical detailed developments and consider only the last two formulas that express the two probabilities.

Let us examine now the quantum neurological connections.

The corresponding brain circuit is just the Mach-Zehnder interferometer (Figure 5).

V1 and the Brodmann area 20 act as 50\% reflector beamsplitters and the secondary visual cortex (V2) and associative visual cortex (V3 - V4) act as $100 \%$ reflector beamsplitters.

Let us explain the reason because we include in the analysis these structures. In humans, to date it has been described that at least two dozen richly interconnected visual areas outside V1 [47] are involved in a dorsal and ventral stream. The first process information about the localization of objects and are mostly found in the posterior/superior part of the brain, and the latter, which forms conscious representations of the identity of objects, are mostly found in the posterior/inferior part of the brain and is the stream we have included in our model.

Although about half of the cortex is involved in visual processing, V1 represents a crucial node as the primary distributor of almost all visual information that reaches other cortical areas. It is possibly the best characterized area of primate cortex also if its direct contribution to conscious visual experience remains still some times controversial. V1 occupies most of the surface of the occipital lobe and its anatomical structure is very similar to the rest of the neocortex: it is divided into six layers distinguished both by the type of neurons that it contains (i.e. stellate, pyramidal cells) and by the connections that it makes with other areas of the brain. This is the area in which the cortex begins to reconstitute the image from the retina, and it exerts profound transformations on the information that comes from the thalamus.

V1 sends a large proportion of connections to neurons located in the secondary visual cortex (V2) that corresponds to Brodmann's area 18 containing a retinotopic mapping of the contralateral visual hemifield. Next, V2 send fibers to V3, mainly found in the same Brodmann's area, and V4, found in the Brodmann's area 19. Both, V3 and V4, are involved in the elaboration of increasingly complex, specialized representations of the outside world. Finally, connections reach Brodmann area 20, part of the fusiform gyrus, which is particularly associated with higher processing of visual stimuli participating in the integration of visual elements into perceptual wholes (single objects). Following such profile neurological level brain creates an awareness of the external world through vision (Figure 6).

In the neurobiology of cognition, there are two main proposals about visual information processing and awareness, considering here visual awareness as the specific contents of consciousness for items in immediate sight. The first one, hierarchical models, establish that our cortex analyzes visual inputs from down to top, that is, at increasing levels of complexity and specificity, being the input accessible to awareness only at higher levels

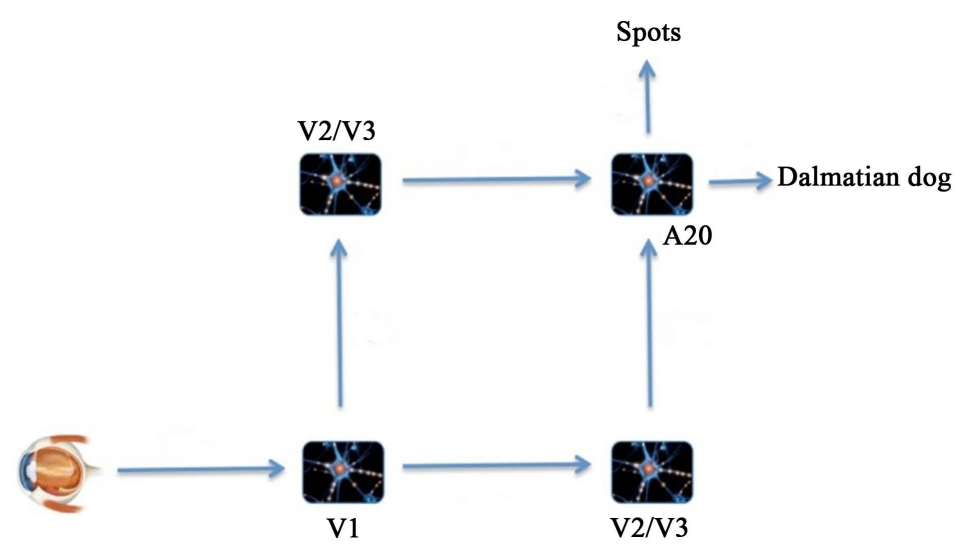

Figure 5. Quantum neurological model. 


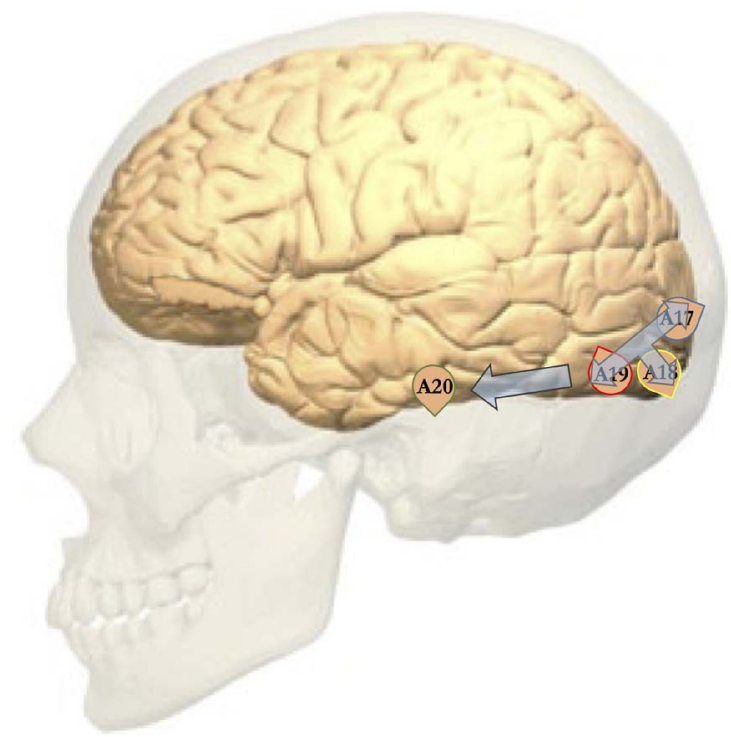

Figure 6. Schematic diagram of connections between a subset of cortical visual areas.

of the visual processing system corresponding to higher levels of visual cortex. It doesn't mean that the neural activity in V1 is insignificant, but implies that it is important only to maintain normal vision and has no function in representing conscious visual information, probably due to its lack of direct projections to prefrontal cortex [48].

Interactive models, meanwhile, proposed that all cortical visual areas are involved directly in visual awareness by forming dynamic recurrent circuits, included V1which through reciprocal connections with many extrastriate areas maintains a visual representation in awareness [49]. The consequence deriving from this approach is that, in these models, the question of what areas are considered top and bottom acquire a new significance; in regard to V1, for example, it exerts veto power over higher areas, modulating their activity and, therefore, having a crucial role in the visual information processing system. The role of V1 in our model follows this criterion: the cortical gate of visual system represents the first profound transformation of the information that, as a last resort, reaches the area 20 to generate a perceptual whole.

Although it has been established that V2, V3 and V4 act as integration areas, in our model we consider that the degree of integration is smaller than that carried out in V1 and in the area 20 and, therefore, we model V2-V3-V4 as a $100 \%$ beams plitters integration process and V1 and area 20 as a $50 \%$ beamsplitters (significant integration process). The theoretical (and quantitative) question derived from the fact that an area is able to act as a beamsplitter is resolved by several neuropsychological studies [50]-[53] that suggest that, for example, in V1 is not necessary to recruit the whole area to generate a percept, but only the activity of a subset of neurons is crucial to the conscious perception of a stimulus, as establishes the dynamic core hypothesis [54], that is to say that the ability to act as a beamsplitters could not be present in all cells.

We can summarize the complete proposed model by the following complete scheme (Figure 7) that integrates the previous one but does not modify our previous formal derivation

This is finally the quantum neurological model that we propose the explanation of the mechanism of the ambiguous figures and in particular the case of the Dalmatian dog.

As repeatedly outlined in the previous sections, one of the ways to analyze how the brain processes visual information is to employ ambiguous figures. We repeat here that these kind of figures have been widely used in research paradigms during the last two centuries and result in rivalry with spontaneous alternation between two percepts, as we can see in the Rubin's face or in the Necker cube, that allows us to conclude that a mental representation of a perceived object is unique even if we may be aware of the possible ambiguity of any given representation [11] [13]-[16] [26] [28]. However, sometimes ambiguity is not presented as mutually exclusive percepts that could alternate under the viewer's eye but one of the percepts prevails over the other, as it happens in the figure of the Dalmatian dog where we can determine the presence of one defined percept (a dog figure) and one undefined (spots) (see Figure 8). 


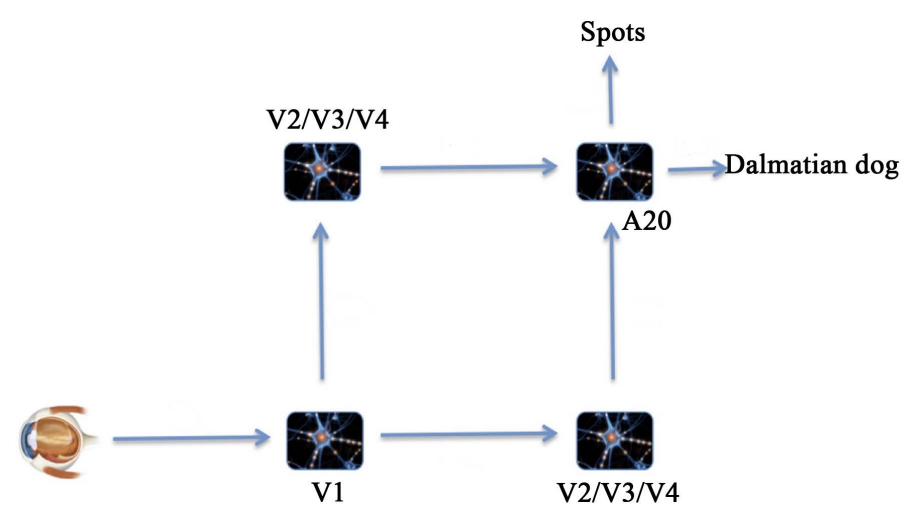

Figure 7. Complete quantum neurological model.

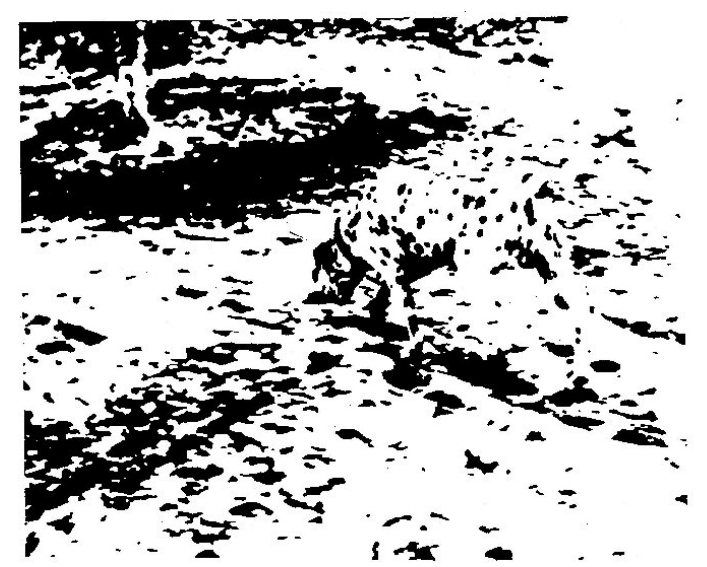

Figure 8. The Dalmatian dog as example of an ambiguous figure.

We intend to outline here also that it has been frequently used by the Gestalt psychology being a clear example of the fact that, although an individual can potentially have multiple representations of a given choice situation, it can attend finally to only one representation at any given time, that is, there is a coexistence of a potential, followed from an actual state of consciousness according to the quantum model that we have elaborated in our studies.

To conclude assuming all our previous studies we have to outline that our Mach-Zenhder interferometer runs at brain level in the following manner. The ambiguous figure gives the material input that is elaborated in the V1; V2/V3/V4; A20 as perceptive-cognitive function that is elaborated according to the rules of quantum mechanics and therefore responding to quantum superposition at the levels of BS splitter and as simple phase of the wave function at the levels of V2, V3 and V4.

Generally speaking we explain the case of the ambiguous figures as well as the particular case of the Dalmatian dog. The probabilities to obtain a final awareness state confined in D1 or D2 are given, as previously derived, by

$$
\operatorname{Prob}(Y=1)=\frac{1}{2}(1-\cos \varphi)
$$

and

$$
\operatorname{Prob}(X=1)=\frac{1}{2}(1+\cos \varphi)
$$

In the case of the ambiguous figures we have values of $\varphi$ that oscillate in the interval $(0, \pi)$ and finally, under the influence of the awareness decay in a given time interval to a value of 0 or to $\pi$. In the case of the Dalmatian dog the decay happens instead systematically to the $\pi$ value. 


\section{Conclusions}

In this paper, we have reassumed the foundations of quantum cognition that we have given starting with our experimental and theoretical studies through the use of ambiguous figures. We have outlined that some basic principles of quantum mechanics as the superposition quantum principle, the collapse of the wave function, the intrinsic indetermination as outlined from this theory and, finally, quantum interference effects, enter in a fundamental manner during the perceptive and cognitive performance in brain dynamics in humans Here we can ask ourselves what is the meaning of Quantum Cognition. It is known that there are many theories about cognition and brain. In addition to those quoted at the beginning, we remember, for instance, the QFT based-study on neuronal collective behaviors [55] [56]. In our work, we used the basic formalism of the non-relativistic QM in Clifford Version. This choice is functional to a simple as well as general message of epistemic nature, i.e. QM is a system theory of contextual information. In particular, QM indicates that in many situations the indifference principle of the system state with respect to an observer, typical of the classic systems, must be given up and we have to adopt an approach that takes into account that the closure relation is only valid for the eingenvalues. It implies a choice that fixes in a global way our knowledge of the system state. We could say that our idea about the ambiguous figures is that of an objective theory of a cognitive "collapse" [57]-[59] for the QM as General Systems Theory and the role of Clifford Algebra see [60].

In order to give further support to our results, in addition to the mention of our previous experimental results, we have introduced those of a new experiment by using ambiguous figures. In our previous experiments, we selected two groups of subjects and to the first group we posed the task on an ambiguous figure, posing only two alternatives, to answer $(+)$ or $(-)$, to the second group we first asked to answer to a submitted second ambiguous figure and soon after to the same ambiguous figure given to the first group. In the case of the present experiment, we modified the structure of the experimentation in the sense that to the second group we presented an ambiguous figure but this time we did not ask to answer but only to look at. Soon after, we gave the subsequent ambiguous figure, asking to the subjects to answer. Also in this last version of the experiment, we verified experimentally the important presence of the quantum interference during perception and cognition of ambiguous figures confirming the elective role of such effect of quantum interference and the fundamental result that, at the level of our awareness, also consciousness responds to the basic rules of quantum mechanics.

Encouraged from such last results, we described a neurological quantum model of perceptive-cognitive functions in ambiguous figures using what we previously introduced in our previous study. In detail, we used a celebrated device of physics that was the Mach-Zehnder interferometer and for the first time we were able to reformulate a whole neurological model developed in strong analogy with the above mentioned device. Our efforts in the present paper were dedicated to the analysis at the neurological level identifying that brain areas V1 and A20 function as BS splitter while instead V2/V3/V4 function as mirrors. The results of our elaboration appear to be of valuable interest because this formulation explains, for the first time, the basic mechanism involved in brain dynamics during perception and cognition of ambiguous figures including also the particular case of the Dalmatian dog.

\section{References}

[1] Naccache, L. (2005) Visual Phenomenal Consciousness: A Neurological Guided Tour. Progress in Brain Research, 150, 185-195. http://dx.doi.org/10.1016/S0079-6123(05)50013-X

[2] Hameroff, S.R., Craddock, T.J. and Tuszynski, J.A. (2014) Quantum Effects in the Understanding of Consciousness. Journal of Integrative Neuroscience, 13, 229-252. http://dx.doi.org/10.1142/S0219635214400093

[3] Tarlaci, S. and Pregnolato, M. (2015) Quantum Neurophysics: From Non-Living Matter to Quantum Neurobiology and Psychopathology. The International Journal of Psychophysiology, In Press.

[4] Hu, H. and Hu, M. (2004) Spin-Mediated Consciousness Theory: Possible Roles of Neural Membrane Nuclear Spin Ensembles and Paramagnetic Oxygen. Medical Hypotheses, 63, 633-646. http://dx.doi.org/10.1142/S0219635214400093

[5] Venkatasubramanian, G. (2015) Understanding Schizophrenia as a Disorder of Consciousness: Biological Correlates and Translational Implications from Quantum Theory Perspectives. Clinical Psychopharmacology and Neuroscience, 13, 36-47. http://dx.doi.org/10.9758/cpn.2015.13.1.36

[6] Craddock, T.J., Priel, A. and Tuszynski, J.A. (2014) Keeping Time: Could Quantum Beating in microtubules Be the Basis for the Neural Synchrony Related to Consciousness? Journal of Integrative Neuroscience, 13, 293-311. 
http://dx.doi.org/10.1142/S0219635214400019

[7] Paraan, M.R., Bakouie, F. and Gharibzadeh, S. (2014) A More Realistic Quantum Mechanical Model of Conscious Perception during Binocular Rivalry. Frontiers in Computational Neuroscience, 8, 15. http://dx.doi.org/10.1142/S0219635214400019

[8] Auffray, C. and Nottale, L. (2008) Scale Relativity Theory and Integrative Systems Biology: 1. Founding Principles and Scale Laws. Progress in Biophysics and Molecular Biology, 97, 79-114; Scale Relativity Theory and Integrative Systems Biology: 2. Macroscopic Quantum-Type Mechanics. Progress in Biophysics and Molecular Biology, 97, 115157.

[9] Nottale, L. (2014) Macroscopic Quantum-Type Potentials in Theoretical Systems Biology. Cells, 1, 1-35. http://dx.doi.org/10.3390/cells3010001

[10] Conte, E. (1983) Exploration of Biological Function by Quantum Mechanics Using Biquaternions. In: Le Concept d'organisation en Cybernétique, Namur, 22-27 Agosto 1983, 16-23, Association Internationale de Cybernètique, Namur.

[11] Conte, E. (2008) Testing Quantum Consciousness. Neuroquantology, 6, 126-139. http://dx.doi.org/10.14704/nq.2008.6.2.167

[12] Conte, E. (2009) A Brief Note on Time Evolution of Quantum Wave Function and of Quantum Probabilities during Perception and Cognition of Human Subjects. NeuroQuantology, 7, 435-448. http://dx.doi.org/10.14704/nq.2009.7.3.243

[13] Conte, E. (2010) On the Possibility That We Think in a Quantum Probabilistic Manner. NeuroQuantology, 8, 3-47. http://dx.doi.org/10.14704/nq.2010.8.4.349

[14] Conte, E. (2011) An Investigation on the Basic Conceptual Foundations of Quantum Mechanics by Using the Clifford Algebra. Advanced Studies in Theoretical Physics, 5, 485-544.

[15] Conte, E. (2011) On the Logical Origins of Quantum Mechanics Demonstrated by Using Clifford Algebra: A Proof That Quantum Interference Arises in a Clifford Algebraic Formulation of Quantum Mechanics. Electronic Journal of Theoretical Physics, 8, 109-126.

[16] Conte, E. (2011) On the Logical Origins of Quantum Mechanics Demonstrated by Using Clifford Algebra. NeuroQuantology, 9, 231-242. http://dx.doi.org/10.14704/nq.2011.9.2.397

[17] Conte, E. (2014) Can Current Quantum Cognition Studies Give Indication on the Manner in Which Human Cognition Arose $a b$ Initio? Psychology, 5, 798-800. http://dx.doi.org/10.4236/psych.2014.58090

[18] Conte, E. (2014) Answer to Giancarlo Ghirardi: Quantum Superpositions and Definite Perceptions: Envisaging New Feasible Experimental Tests. A Novel Proposal for Quantum Mechanics, Perception and Cognitive Science? International Journal of Theoretical Physics, 54, 672-679. http://dx.doi.org/10.1007/s10773-014-2259-6

[19] Conte, E. (2015) Additional Comments Added to Our Recent Answer to G. Ghirardi. Journal of Modern Physics, 6, 12-15. http://dx.doi.org/10.4236/jmp.2015.61002

[20] Conte, E. (2015) What Path Monitor: A Brief Note on Quantum Cognition and Quantum Interference, the Role of the Knowledge Factor. Psychology, 6, 291-296. http://dx.doi.org/10.4236/psych.2015.63029

[21] Conte, E. (2015) A Brief Comment on Some Recent Evaluations by Basieva and Khrennikov, Wang et al., BoyerKassem et al., on Order Effects in Quantum Cognition. NeuroQuantology, 13, 250-252. http://dx.doi.org/10.14704/nq.2015.13.2.821

[22] Conte, E. (2015) On Some Explanations and Analysis of the Basic Foundations of Quantum Cognition: Comments on a Paper by Pothos, Busemeyer and Trueblood. NeuroQuantology, 13, 371-383. http://dx.doi.org/10.14704/nq.2015.13.3.866

[23] Conte, E., Todarello, O., Federici, A., Vitiello, F., Lopane, M. and Khrennikov, A.Y. (2004) A Preliminary Evidence of Quantum Like Behaviour in Measurements of Mental States. In: Khrennikov, A.Y., Ed., Quantum Theory: Reconsideration of Foundations 2, Vaxjo University Press, Vaxjo, 679-702.

[24] Conte, E., Pierri, G., Mendolicchio, L., Khrennikov, A.Y. and Zbilut, J.P. (2006) On Some Detailed Examples of Quantum Like Structures Containing Quantum Potential States in the Sphere of Biological Dynamics. Cornell University Library, Ithaca. arXiv:physics/0608236.

[25] Conte, E., Khrennikov, A.Y. and Zbilut, J.P. (2006) The Transition from Ontic Potentiality to Actualization of States in Quantum Mechanical Approach to Reality: The Proof of a Mathematical Theorem to Support It. Cornell University Library, Ithaca. arXiv:quant-ph/0607196.

[26] Conte, E., Todarello, O., Federici, A., Vitiello, F., Lopane, M., Khrennikov, A.Y. and Zbilut, J.P. (2007) Some Remarks on an Experiment Suggesting Quantum-Like Behaviour of Cognitive Entities and Formulation of an Abstract Quantum Mechanical Formalism to Describe Cognitive Entity and Its Dynamics. Chaos, Solitons and Fractals, 31, 1076-1088. http://dx.doi.org/10.1016/j.chaos.2005.09.061 
[27] Conte, E., Khrennikov, A.Y., Todarello, O., Federici, A. and Zbilut, J.P. (2008) A Preliminary Experimental Verification on the Possibility of Bell Inequality Violation in Mental States. NeuroQuantology, 6, 214-221. http://dx.doi.org/10.14704/nq.2008.6.3.178

[28] Conte, E., Khrennikov, A.Y., Todarello, O., Federici, A., Mendolicchio, L. and Zbilut, J.P. (2009) Mental States Follow Quantum Mechanics during Perception and Cognition of Ambiguous Figures. Open Systems and Information Dynamics, 16, 85-100. http://dx.doi.org/10.1142/S1230161209000074

[29] Conte, E., Khrennikov, A.Y., Todarello, O., Federici, A. and Zbilut, J.P. (2009) On the Existence of Quantum Wave Function and Quantum Interference Effects in Mental States: An Experimental Confirmation during Perception and Cognition in Humans. NeuroQuantology, 7, 204-212. http://dx.doi.org/10.14704/nq.2009.7.2.225

[30] Conte, E., Todarello, O., Laterza, V., Khrennikov, A.Y., Mendolicchio, L. and Federici, A. (2010) A Preliminary Experimental Verification of Violation of Bell Inequality in a Quantum Model of Jung Theory of Personality Formulated with Clifford Algebra. Journal of Consciousness Exploration \& Research, 1, 831-849.

[31] Conte, E., Altamura, M., De Salvia, M., Federici, A., Bellomo, A. and Zbilut, J.P. (2011) On a New Form of Chaos Based on Deterministic Chaos plus Added Quantum Mechanical Components: The Perspectives of Application in Cognitive Processes in Psychology. Chaos and Complexity Letters, 5, 27-59.

[32] Conte, E., Khrennikov, A.Y., Todarello, O., De Robertis, R., Federici, A. and Zbilut, J.P. (2011) On the Possibility That We Think in a Quantum Mechanical Manner: An Experimental Verification of Existing Quantum Interference Effects in Cognitive Anomaly of Conjunction Fallacy. Chaos and Complexity Letters, 4, 123-136.

[33] Conte E. and Lucas, R.F. (2015) First Time Demonstration of the Quantum Interference Effect during Integration of Cognition and Emotion in Children. World Journal of Neuroscience, 5, 91-98. http://dx.doi.org/10.4236/wjns.2015.52011

[34] Conte, E. (2010) A Proof of Von Neumann’s Postulate in Quantum Mechanics. In: Khrennikov, A.Y., Ed., Quantum Theory Reconsideration of Foundations 5, American Institute of Physics, New York, 201-205. http://dx.doi.org/10.1063/1.3431489

[35] Conte, E., Santacroce, N. and Federici, A. (2012) A Possible Quantum Model of Consciousness Interfaced with a NonLipschitz Chaotic Dynamics of Neural Activity (Part I) and (Part II). Journal of Consciousness Exploration and Research, 3, 905-936.

[36] Conte, E., Todarello, O., Federici, A., Santacroce, N., Laterza, V. and Khrennikov, A.Y. (2012) May We Verify NonExisting Dispersion Free Ensembles by Application of Quantum Mechanics in Experiments at Perceptive and Cognitive Level? Neuroquantology, 10, 14-19. http://dx.doi.org/10.14704/nq.2012.10.1.510

[37] Conte, E., Khrennikov, A.Y., Todarello, O., De Robertis, R., Federici, A. and Zbilut, J.P. (2012) On the Possibility That We Think in a Quantum Mechanical Manner: An Experimental Verification of Existing Quantum Interference Effects in Cognitive Anomaly of Conjunction Fallacy. In: On the Borders of Complexity, Nova Science Publishers, Inc., Hauppauge, 149-163.

[38] Conte, E., Santacroce, N., Laterza, V., Conte, S., Federici, A. and Todarello, O. (2012) The Brain Knows More than It Admits: A Quantum Model and Its Experimental Confirmation. Electronic Journal of Theoretical Physics, 9, 72-110.

[39] Jansen, F.K. (2015) Quantum Mechanics Is Not Physical Reality but Mental Potentiality because of the Law of NonContradiction. Neuroquantology, 13, 1-10. http://dx.doi.org/10.14704/nq.2015.13.1.802

[40] Eccles, J.C. (1986) Do Mental Events Cause Neural Events Analogously to the Probability Fields of Quantum Mechanics? Proceedings of the Royal Society of London. Series B, Biological Sciences, 227, 411-428. http://dx.doi.org/10.1098/rspb.1986.0031

[41] Margenau, H. (1984) The Miracle of Existence. Ox Bow Press, Woodbridge.

[42] Hecht, S., Shlaer, S. and Pirenne, M.H. (1942) Energy, Quanta, and Vision. Journal of General Physiology, 25, 819840. http://dx.doi.org/10.1085/jgp.25.6.819

[43] Baylor, D.A., Lamb, T.D. and Yau, K.W. (1979) The Membrane Current of Single Rod Outer Segments. The Journal of Physiology, 288, 589-611. http://dx.doi.org/10.1016/0042-6989(79)90077-4

[44] Woo, C.H. (1981) Consciousness and Quantum Interference: An Experimental Approach. Foundations of Physics, 11, 933-943. http://dx.doi.org/10.1007/BF00727109

[45] Conte, E. (2013) A Clifford Algebraic Analysis Gives Mathematical Explanation of Quantization of Quantum Theory and Delineates a Model of Quantum Reality in Which Information, Primitive Cognition Entities and a Principle of Existence Are Intrinsically Represented ab Initio. World Journal of Neuroscience, 3, 157-170. http://dx.doi.org/10.4236/wjns.2013.33021

[46] Conte, E. (2012) Advances in Application of Quantum Mechanics in Neuroscience and Psychology: A Clifford Algebraic Approach. Nova Science Publishers, New York.

[47] Felleman, D.J. and Van Essen, D.C. (1991) Distributed Hierarchical Processing in the Primate Cere-Bralcortex. Cere- 
bral Cortex, 1, 1-47. http://dx.doi.org/10.1093/cercor/1.1.1

[48] Crick, F. and Koch, C. (1995) Are We Aware of Neural Activity in Primary Visual Cortex? Nature, 375, 121-123. http://dx.doi.org/10.1038/375121a0

[49] Tong, F. (2003) Primary Visual Cortex and Visual Awareness. Nature Reviews Neuroscience, 4, 219-229. http://dx.doi.org/10.1038/nrn1055

[50] Leopold, D.A. and Logothetis, N.K. (1996) Activity Changes in Early Visual Cortex Reflect Monkeys’ Percepts during Binocular Rivalry. Nature, 379, 549-553. http://dx.doi.org/10.1038/379549a0

[51] Sheinberg, D.L. and Logothetis, N.K. (1997) The Role of Temporal Cortical Areas in Perceptual Organization. Proceedings of the National Academy of Sciences of the United States of America, 94, 3408-3413. http://dx.doi.org/10.1073/pnas.94.7.3408

[52] Tononi, G., Srinivasan, R., Russell, D.P. and Edelman, G.M. (1998) Investigating Neural Correlates of Conscious Perception by Frequency-Tagged Neuromagnetic Responses. Proceedings of the National Academy of Sciences of the United States of America, 95, 3198-3203. http://dx.doi.org/10.1073/pnas.95.6.3198

[53] Srinivasan, R., Russell, D.P., Edelman, G.M. and Tononi, G. (1999) Increased Synchronization of Neuromagnetic Responses during Conscious Perception. Journal of Neuroscience, 19, 5435-5448.

[54] Tononi, G. and Edelman, G.M. (1998) Consciousness and Complexity. Science, 282, 1846-1851. http://dx.doi.org/10.1126/science.282.5395.1846

[55] Ricciardi, L.M. and Umezawa, H. (1967) Brain Physics and Many-Body Problems. Kibernetik, 4, 44-48. http://dx.doi.org/10.1007/BF00292170

[56] Vitiello, G., Freeman, W.J. (2008) The Dissipative Quantum Model of Brain and Laboratory Observations. In: Licata, I. and Sakaji, A., Eds., Physics of Emergence and Organization, World Scientific, Singapore City, 233-251.

[57] Heylighen, F. (1990) Classical and Non-Classical Representations in Physics II: Quantum Mechanics. Cybernetics and Systems, 21, 477-502. http://dx.doi.org/10.1080/01969729008902255

[58] Orlov, Y. (1994) The Logical Origins of Quantum Mechanics. Annals of Physics, 234, 245-259. http://dx.doi.org/10.1006/aphy.1994.1081

[59] Licata, I. (2008) Emergence and Computation at the Edge of Classical and Quantum Systems. In Licata, I. and Sakaji, A., Eds., Physics of Emergence and Information, World Scientific, Singapore City, 1-25. http://dx.doi.org/10.1142/9789812779953_0001

[60] Caianello, E.R. (1992) Quantum and Other Physics as Systems Theory. La Rivista del Nuovo Cimento, 15, 1-65. http://dx.doi.org/10.1007/BF02743060 